\title{
Mortality rates for giant aneurysms
}

\author{
Zakaria Hakma • Raghu Ramaswamy • \\ Christopher Miranda Loftus
}

Received: 4 April 2011 / Accepted: 5 April 2011 /Published online: 15 May 2011

(C) Springer-Verlag 2011

In this issue, Szmuda and coworkers present a series of internal carotid artery (ICA) (only) aneurysms, all treated with open surgery, in which there is no material difference in morbidity and mortality figures between routine small aneurysms and giant aneurysms. This finding is in contradistinction to previous surgical reports and accepted surgical teachings, and this variance merits our further consideration here.

Giant intracranial aneurysms (GIAs) - defined as those greater than $2.5 \mathrm{~cm}$ in diameter-often pose a unique and challenging problem in their microsurgical or endovascular treatment. They are relatively infrequent, comprising approximately $5 \%$ of all aneurysms in most series $[2,4]$.

The size definition $(>2.5 \mathrm{~cm})$ came from Locksley's pivotal work that classified the aneurysms according to size and their rate of morbidity and mortality [7].

Giant aneurysms are dangerous. The mortality rates for untreated giant aneurysms have been reported to be as high as 65 and $100 \%$ after $2-5$ years of follow-up [5, 9], with a rupture rate for conservatively treated intradural giant aneurysms roughly around $8-10 \%$ per year [10].

Morphologically, giant aneurysms are divided into saccular and fusiform types, with fusiform aneurysms arising more commonly in the vertebrobasilar and middle cerebral territories [1].

The treatment of GIAs, like smaller aneurysms, is generally directed toward complete exclusion of the lesion from the circulation, with preservation of flow in all parent arteries.

Z. Hakma $\cdot$ R. Ramaswamy $\cdot$ C. M. Loftus $(\bowtie)$

Department of Neurosurgery, Temple University,

3401 N. Broad Street, Parkinson C-540,

Philadelphia, PA 19140, USA

e-mail: cloftus@temple.edu
This can be accomplished with direct clip reconstruction, aneurysm trapping with or without surgical bypass, acute proximal vessel occlusion with or without surgical bypass, and Selverstone clamping (delayed proximal occlusion). The reported modern surgical mortality rates for the treatment of both ruptured and unruptured giant aneurysms vary between $6 \%$ and $22 \%[3,6]$

From an endovascular perspective, these lesions are often not favorable because they frequently have broad necks, distorting the anatomy of the parent vessel, incorporating branch arteries at the base, and there is a high incidence of intraluminal thrombosis. These anatomic factors make giant aneurysms difficult to coil and obliterate completely, leading to residual or recurrent aneurysms, multiple retreatments, occasional rehemorrhages, and neurological deterioration from progressive aneurysm enlargement.

Current endovascular techniques (coil embolization with or without balloon assistance, neck remodeling with stents, liquid embolics, etc.) for the treatment of giant cerebral aneurysms still have significant periprocedural risks for the patients. These risks are only compounded by the severity of their cerebral vascular pathology, and many times are not satisfactory in terms of the long-term durability of aneurysm occlusion.

Flow diversion approaches to intracranial aneurysm treatment have offered many promising early results; but some apparently successful treatments have been complicated by later aneurysm hemorrhage. Combined microsurgical and endovascular strategies have been used for more than a decade to treat complex intracranial aneurysms with varying degrees of success [8].

Szmuda and Sloniewski, in this issue, report similar mortality rates, short-term outcomes, and long-term outcomes after surgery for giant and smaller ICA aneurysms. 
They did not offer their patients an endovascular option during this study period. In a retrospective review of 328 ICA aneurysms with a high fraction of "giant" lesions (78 giant, 250 smaller), 242 were ruptured and 86 were unruptured. Hunt and Hess scores, Fisher grades, Glasgow Coma Scale (GCS), and World Federation of Neurological Surgeons (WFNS) scores did not differ between giant and smaller aneurysms. There were no significant differences in complication rates between these two groups and the mortality rates were similar. In regards to the occurrence of complications in the short-term, three independent risk factors were found: general complications in the giant aneurysm group, Fisher grade 4 status, and the presence of a new postoperative neurologic deficit following surgery of smaller aneurysms. The long-term outcome after surgery was influenced by factors of age and of concomitant disease.

The authors espouse that while surgery of giant aneurysms is generally felt - as discussed above - to be more dangerous than for smaller aneurysms, complication rates - assessed in their series by short-term and long-term outcome - can be similar between the two groups.

As we mentioned, this is a single institution, single surgeon experience, and while excellent and provocative, it is not reflective of the experience of the neurosurgical community as a whole.

Limitations of this study include the absence of the endovascular option, as none of these patients were offered an endovascular procedure. The authors were not in a position to offer newer technologies for these challenging aneurysms (especially flow diversion with Pipeline or SILK that might be a viable option for some of these unruptured aneurysms).

The timing of surgery after rupture was not presented. Timing of surgery is an important factor when discussing surgical results, to eliminate selection bias and bring into focus the crossing lines of surgical morbidity (higher with early surgery) versus rebleeding and management morbidity (higher with delayed surgery). Timing of surgery also affects the ability to aggressively treat vasospasm, depending on the presence of a secured versus an unsecured aneurysm.

In this study, many patients were lost to follow-up; in fact, in the group of patients with unfavorable short-term outcome [Glasgow Outcome Scale (GOS) grade 2 or 3], the authors collected SF-36 queries from 7\%, while the quality of life was assessed in $73.5 \%$ of patients with good shortterm outcomes. This creates an issue for interpreting the long-term outcomes and assessing the rate of rebleeding after surgical treatment.

Let us consider the differences in populations that may explain this inability to assign higher perioperative risk to giant ICA aneurysms. First, giant aneurysms in this series were less likely to present with subarachnoid hemorrhage
(SAH) ( $46 \%$ of giant lesions, $83 \%$ of smaller ones), and we feel that SAH portends a higher treatment morbidity. Second, $15 \%$ of giant lesions were intracavernous, but only $0.8 \%$ of smaller lesions, and the treatment in most cases was flow diversion with high-flow bypass $(9 / 78$ or $12 \%$ of giant aneurysms, only $2 / 250$ or $0.8 \%$ of smaller aneurysms); once again direct clipping is most likely associated with a higher perioperative risk than flow diversion techniques. These considerations skew the risk away from the giant population, and may lull us into a false sense of security that GIA morbidity approaches that of smaller lesions, contrary to conventional surgical dogma.

Table 2 is lucid, and explains clearly that alternatives to direct clipping, probably less morbid in nature, were used in $20 \%$ more of the GIA cases. Indirect treatment proved to be an excellent strategy, as there was no rebleeding in the series, and we are not critical of this, but we do feel that it lowered the risk profile in the GIA group.

While we do not dispute the results here in any way, we recognize that these results are at variance with results of highly experienced neurovascular surgeons and we caution to generalize these results yet; we encourage surgeons to continue to report their results on this challenging disease. We believe that the authors' statistics are credible and valid, and that their conclusions are reasonable considering the data provided. We believe that the variance with accepted teaching is best explained by the group differences between $\mathrm{SAH}$ rate, aneurysm location, and direct versus indirect approach. We would also be curious to know if the authors' experience with posterior circulation GIAs could reproduce these results?

This is an interesting article by experienced surgeons and a worthwhile contribution that challenges the notion of unfavorable giant ICA aneurysm treatment results, and adds to the knowledge base of the treatment of this difficult condition.

Conflicts of interest None.

\section{References}

1. Barrow DL, Alleyne C (1995) Natural history of giant intracranial aneurysms and indications for intervention. Clin Neurosurg 42:214-244

2. Drake CG (1979) Giant intracranial aneurysms: experience with surgical treatment in 174 patients. Clin Neurosurg 26:12-95

3. Gewirtz RJ, Awad IA (1996) Giant aneurysms of the anterior circle of Willis: management outcome of open microsurgical treatment. Surg Neurol 45:409-421

4. Hosobuchi Y (1979) Direct surgical treatment of giant intracranial aneurysms. J Neurosurg 51:743-756 
5. Kodama N, Suzuki J (1982) Surgical treatment of giant aneurysms. Neurosurg Rev 5:155-160

6. Lawton MT, Spetzler RF (1995) Surgical management of giant intracranial aneurysms: experience with 171 patients. Clin Neurosurg 42:245-266

7. Locksley HB (1966) Natural history of subarachnoid hemorrhage, intracranial aneurysms and arteriovenous malformations. Based on 6368 cases in the cooperative study. J Neurosurg 25:219-239

8. Mizoi K, Takahashi A, Yoshimoto T, Fujiwara J, Koshu K (1993) Combined endovascular and neurosurgical approach for paraclinoid internal carotid artery aneurysms. Neurosurgery 33:986-992
9. Peerless SJ, Wallace MC, Drake CG (1990) Giant intracranial aneurysms. In: Youmans JR (ed) Neurological surgery: a comprehensive reference guide to diagnosis and management of neurological problems. Saunders, Philadelphia, pp 1742-1763

10. Wiebers DO, Whisnant JP, Huston J 3rd, Meissner I, Brown RD Jr, Piepgras DG, Forbes GS, Thielen K, Nichols D, O'Fallon WM, Peacock J, Jaeger L, Kassell NF, Kongable-Beckman GL, Torner JC; International Study of Unruptured Intracranial Aneurysms Investigators (2003) Unruptured intracranial aneurysms: natural history, clinical outcome and risks of surgical and endovascular treatment. Lancet 362:103-110 